\title{
Sedation in a child with Klippel- Feil syndrome scheduled for magnetic resonance imaging
}

\author{
Swati Chhabra ${ }^{1}$, S K Singhal ${ }^{2}$, Sadik Mohammed ${ }^{1}$, Ghansham Biyani ${ }^{3}$, and \\ Rakesh Pandey ${ }^{4}$ \\ ${ }^{I}$ Department of Anesthesiology, All India Institute of Medical Sciences, Jodhpur, ${ }^{2}$ Pt. BD Sharma Post Graduate \\ Institute of Medical Sciences, Rohtak, ${ }^{3}$ Axon Anesthesia Associates, Hyderabad, ${ }^{4}$ Department of Anesthesiology, \\ Vardaan Nursing Home \& Trauma Center, Palwal, India
}

Anesthesia or sedation is required in magnetic resonance imaging (MRI) suites to perform imaging in children and uncooperative adults. The choice of anesthesia may range from moderate sedation to general anesthesia depending on the patient's characteristics and/or institutional protocols. Due to challenges in accessing patients in the MRI suite, the anesthetic technique should be chosen carefully. This is even more important when the patient has an anticipated difficult airway.

A four-year-old male child weighing $14 \mathrm{~kg}$ presented with delayed developmental milestones, diminished hearing, and no organized speech. The patient had a diagnosis of Klippel-Feil syndrome (KFS). Ultrasonography of the abdomen and echocardiography ruled out any associated systemic defects and the patient was scheduled for MRI of the brain and cervical spine for further evaluation. After a failed attempt at sedating the patient with oral chloral hydrate in the MRI suite, he was scheduled for MRI under general anesthesia.

The pre-anesthetic evaluation showed that the patient had a short webbed neck with limited extension and a low posterior hairline (Fig. 1). Due to an anticipated difficult airway and the diagnostic nature of the procedure, we planned to provide seda-

Corresponding author: Swati Chhabra, M.D.

Department of Anesthesiology, All India Institute of Medical Sciences, Basni Industrial Area phase 2, Jodhpur 342005, India

Tel: 91-946-8090819, Fax: 91-291-2740531

Email: swati_virgo83@yahoo.co.in

ORCID: https://orcid.org/0000-0002-1718-0330

Received: July 3, 2017.

Revised: July 4, 2017.

Accepted: July 10, 2017.

Korean J Anesthesiol 2018 April 71(2): 163-164

https://doi.org/10.4097/kjae.2018.71.2.163 tion with dexmedetomidine via a backup laryngeal mask airway if required. Intravenous access was obtained with a 22-gauge cannula after application of a eutectic mixture of local anesthetics. Baseline vitals (electrocardiography [ECG], blood pressure, and pulse oxygen saturation $\left[\mathrm{SpO}_{2}\right]$ ) were recorded. A loading dose of $1 \mu \mathrm{g} / \mathrm{kg}$ dexmedetomidine was administered over 10 minutes followed by an infusion of $0.7 \mu \mathrm{g} / \mathrm{kg} / \mathrm{h}$ dexmedetomidine. Monitoring of the depth of sedation was performed based on the Ramsay Sedation Score and the patient was moved onto the MRI table once a score of 5 was achieved; following this, ear plugs were applied. Oxygen supplementation was achieved with fraction of inspired oxygen of 0.28 with continuous monitoring of ECG, noninvasive blood pressure, $\mathrm{SpO}_{2}$, and end-tidal carbon dioxide throughout the diagnostic procedure, which lasted approximately 50 minutes. All of the above parameters were within normal limits and no additional intervention was required.

At the end of the procedure, dexmedetomidine infusion was stopped and the patient was responsive to verbal stimuli after 7 minutes. The patient was discharged home once the discharge criteria were met.

KFS is an inherited condition with the classic triad of a short webbed neck, limited neck movements, and a low posterior hairline [1,2]. KFS may be associated with conductive or sensorineural deafness, congenital heart disease (most commonly a ventricular septal defect), cleft palate, rib defects, and scoliosis. Anesthetic challenges include a difficult airway, cervical spine instability, and associated cardiovascular and genitourinary system abnormalities. There is an increased risk of spinal cord injury during maneuvers such as laryngoscopy, intubation, and placing the patient in an appropriate position for the procedure [3].

Patients with KFS may need to be anesthetized for diagnostic procedures, surgical correction of congenital defects, or any oth-

(c) This is an open-access article distributed under the terms of the Creative Commons Attribution Non-Commercial License (http://creativecommons.org/ licenses/by-nc/4.0/), which permits unrestricted non-commercial use, distribution, and reproduction in any medium, provided the original work is properly cited. 


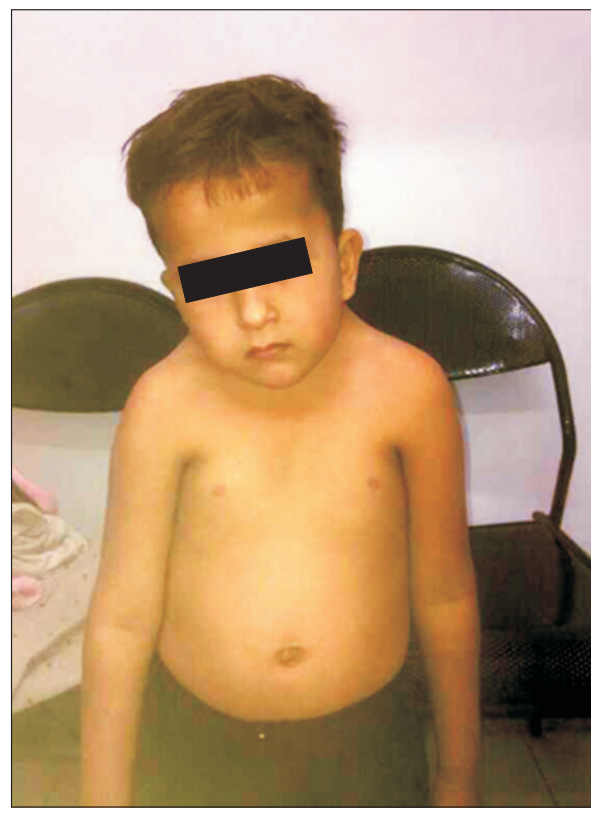

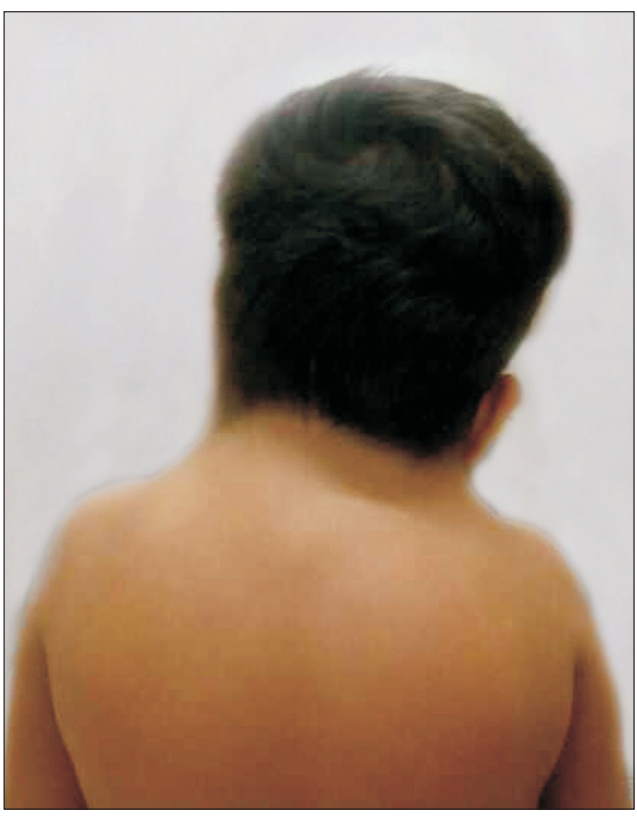

Fig. 1. Our Klippel-Feil syndrome patient. (A) Note the short webbed neck and (B) low posterior hairline. er incidental surgery [4]. An appropriate anesthetic technique should be chosen in consideration of a difficult airway, unstable cervical spine, and any associated systemic abnormality. Two previous reports are available in which children with KFS were scheduled to undergo MRI. In both instances, a laryngeal mask airway was used as an airway conduit for inhalational anesthesia with spontaneous respiration [5].

By using dexmedetomidine for sedation in our patient, we avoided airway manipulation. This was important in view of the difficult airway and cervical spine instability. Care was taken to maintain the head and neck in a neutral position while shifting the patient onto, and from, the MRI table. An adequate level of sedation was achieved without respiratory depression or hemodynamic changes. Thus, dexmedetomidine may be a safe option for sedation in children with KFS without the need for airway manipulation, which can be challenging in these cases.

\section{References}

1. Naguib MG, Maxwell RE, Chou SN. Identification and management of high-risk patients with Klippel Feil syndrome. J Neurosurg 1984; 61: 523-30.

2. Jones KL, Smith S. Recognizable patterns of human malformation. 5th ed. Philadelphia, WB Saunders Company. 1997, pp 320-33.

3. Daum RE, Jones DJ. Fibreoptic intubation in Klippel-Feil syndrome. Anaesthesia 1988; 43: 18-21.

4. Naguib M, Farag H, Ibrahim A el-W. Anaesthetic considerations in Klippel-Feil syndrome. Can Anaesth Soc J 1986; 33: 66-70.

5. Manivel S, Prasad R, Jacob R. Anesthetic management of a child with Klippel-Feil syndrome in the radiology suite. Paediatr Anaesth 2005; 15: $171-2$ 